\title{
Training in computational skills
}

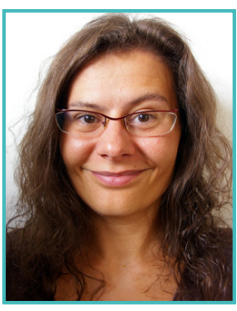

\section{Aleksandra Pawlik}

University of Manchester, Manchester, United Kingdom

Pawlik A (2014) EMBnet.journal 20(Suppl A), e775. http://dx.doi.org/10,14806/ej.20. A.775

This talk will outline the essentials of training in as version control. The skills should scale up enacomputational skills in bioinformatics and be- bling researchers to use large computational reyond. The need for such training is clear: research- sources and cloud infrastructure such as Amazon ers need to be able to work independently and EC2 or Microsoft Azure. The talk will also discuss efficiently using a variety of computational tools. how much of the training in computational skills The skills which they require include the ability to is common across biosciences, and how and automate tasks and build reproducible research when it needs to be adjusted for the particular pipelines, understand and be able to apply good programming practices in a programming language of choice, as well as being familiar with those software engineering tools that provide relevant support for computational research, such purposes of different disciplines. The example of the successful model of Software Carpentry' training shows that building on a common curriculum base makes possible to develop and deliver useful training packages.

1 software-carpentry.org 